\title{
LOS RECURSOS DIGITALES Y SU IMPACTO EN EL DESARROLLO DE LA CAPACIDAD DE ANÁLISIS DE LOS ESTUDIANTES UNIVERSITARIOS
}

\author{
AUTORES: Grace Liliana Figueroa Morán ${ }^{1}$ \\ Julio Pedro Paladines Morán² \\ José Nevardo Paladines Morán ${ }^{3}$
}

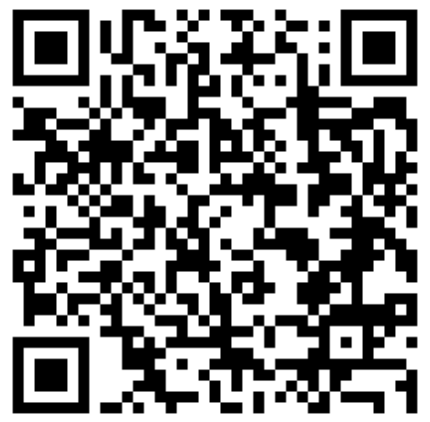

\section{DIRECCIÓN PARA CORRESPONDENCIA:grace.figueroa@unesum.edu.ec}

Fecha de recepción: 31/12/2019

Fecha de aceptación: 21/01/2020

RESUMEN

La nueva sociedad del conocimiento propone una educación más competitiva a través de la aplicación de nuevas herramientas educativas, que favorezcan el proceso de enseñanza y aprendizaje. De entre todas son los recursos digitales los que, a través de una adecuada aplicación, permitirán motivar al estudiante en el momento de crear un nuevo conocimiento. El presente estudio analiza los resultados del uso de los recursos digitales en el desarrollo de la capacidad de análisis de los estudiantes universitarios del primer semestre de la carrera de Ingeniería en Computación y Redes de la Universidad Estatal del Sur de Manabí (UNESUM). El estudio sigue un diseño cuasi-experimental, la muestra estuvo compuesta por 84 alumnos, distribuidos en grupo experimental y de control. Para evaluar el desarrollo de la competencia de análisis de los estudiantes, se utilizaron recursos creados mediante herramientas de autor para la asignatura de Introducción a la informática. Los resultados obtenidos mostraron un incremento significativo en el grupo

\footnotetext{
${ }^{1}$ Licenciada en Ciencia de la Educación-Informática, Magister en Informática Empresarial., Magister en Docencia Universitaria e Investigación Educativa, Especialista en Redes y Comunicaciones de Datos, Diplomado en Autoevaluación y Acreditación Universitaria. Docente de la carrera de Tecnologías de la Información en la Universidad Estatal del Sur de Manabí, ubicada en el Km 1,5, vía a Noboa. Jipijapa, Manabí y Ecuador.

2 (Ingeniero en Sistema, Magister en Informática Empresarial., Especialista en Redes y Comunicaciones de Datos, Diplomado en Autoevaluación y Acreditación Universitaria. Docente de la carrera de Tecnologías de la Información en la Universidad Estatal del Sur de Manabí. Jipijapa, Manabí y Ecuador.

${ }^{3}$ Ingeniero en Sistema, Magister en Informática Empresarial, Magister en Docencia Universitaria e Investigación Educativa, Especialista en Redes y Comunicaciones de Datos, Diplomado en Autoevaluación y Acreditación Universitaria. Docente de la carrera de Tecnologías de la Información en la Universidad Estatal del Sur de Manabí. Jipijapa, Manabí y Ecuador.
} 
Grace Liliana Figueroa Morán, Julio Pedro Paladines Morán, José Nevardo Paladines Morán...

experimental, con lo que se comprobó que el uso de los recursos digitales mejora la capacidad de análisis de los estudiantes.

PALABRAS CLAVE: Capacidad de análisis, herramientas educativas, proceso enseñanza aprendizaje, recursos digitales.

\section{DIGITAL RESOURCES AND THEIR IMPACT ON THE DEVELOPMENT OF THE ANALYSIS CAPACITY OF UNIVERSITY STUDENTS}

\section{ABSTRACT}

The new knowledge society proposes a more competitive education through the application of new educational tools that favor the teaching and learning process. One of those tools is the digital resources that, through an appropriate application, allows the student to be motivated when creating a new knowledge. The present study analyzes the results of the use of digital resources in the development of the analysis capacity of the university students of the first semester of the grade Computer and Network Engineering of the Universidad Estatal del Sur de Manabí (UNESUM). The study follows a quasi-experimental design, the sample was composed of 84 students, distributed in experimental group and control group. To assess the development of the students' analysis competence, resources created in several authoring tools were used for the subject of Introduction to Computer Science. The results obtained showed a significant increase in the experimental group, which proved that the use of digital resources improves the students' analysis capacity.

KEYWORDS: Analysis capacity, Educational tools, teaching-learning process, digital resources.

\section{INTRODUCCIÓN}

Actualmente la tecnología y particularmente la Internet, se ha convertido en un medio potencial que ofrece un sinnúmero de recursos digitalizados en línea, que inicialmente fueron creados para diversas actividades, pero que con el transcurso del tiempo se han convertido en herramientas potenciales para ser puestas en práctica en el ámbito educativo (Bautista, Borges, y Forés, 2006). Esto ha conllevado a que las instituciones educativas y en particular las universidades realicen cambios tanto metodológicos como estratégicos en la educación, para poder estar acorde con la realidad actual (Salinas Ibáñez, 2004).

La incorporación de tecnologías en la docencia es una práctica cada vez más frecuente en el ámbito universitario (Isusi, 2018). Esto supone un reto para el docente, ya que debe revisar el currículo de las asignaturas e incorporar estos instrumentos para beneficiar el proceso de aprendizaje.

Las TIC son una de las tantas herramientas que un docente puede utilizar para favorecer el proceso de enseñanza aprendizaje, pero para hacerlo y generar un impacto positivo en el estudiante, el profesor debe utilizar el concepto de ellas y el propósito de utilizarlas en el aula. Con relación a ello, Trigueros, Sánchez y Vera (2012) señalan que las TIC permiten que el docente sea más 
receptivo a los cambios en la metodología y en su rol en cuanto a la orientación, dinamización de grupos, motivación de los estudiantes, diseño y gestión de los entornos de aprendizaje, creación de recursos y evaluación formativa (Clutterbuck, 2015).

Otro de los elementos esenciales en la implementación de las TIC en los centros educativos, es la organización y la cultura tradicionales de la escuela, es decir, la dificultad que encuentran los procesos de innovación con TIC al intentar romper o transformar las asentadas normas del centro escolar. En este contexto, resulta complicado producir los grandes cambios que auguraba la integración de las TIC en las aulas (Medina y Ballano, 2015).

Por lo tanto, para que un docente pueda ser un gestor activo de ambientes de aprendizajes enriquecidos por TIC, debe conocer el uso de cada una de estas herramientas y estar en constante capacitación debido a que la sociedad evoluciona rápidamente y es necesario que los docentes vayan en la misma línea (Salinas, 2004).

En ese sentido, en este trabajo se presenta una conceptualización, características y consideraciones pedagógicas, que se debe tomar en cuenta en el momento de utilizar un recurso digital, incluso se enfocan otros aspectos relacionados con la temática para finalmente llegar a conocer el aporte del uso de los recursos digitales en el aprendizaje de los estudiantes universitarios (Cruz, 2017).

\section{DESARROLLO}

\section{Recursos digitales}

Los recursos digitales o también llamados recursos educativos digitales (RED) están diseñados para favorecer algunas actividades relativas al proceso de aprendizaje, son materiales compuestos por medios digitales y producidos con el fin de facilitar el desarrollo de las actividades de aprendizaje (Zapata, 2012).

Los recursos digitales ofrecen nuevas oportunidades en los procesos de enseñanza al incorporar la imagen, el sonido y la interactividad, como elementos que refuerzan la comprensión y motivación de los estudiantes (Mondragón, 2014).

También apoyan el aprendizaje de los estudiantes y el aumento de su éxito, por eso su importancia, puesto que pueden aumentar el logro estudiantil (AUTOR). Por otra parte, es importante considerar siempre el aprendizaje de los estudiantes como centro de interés más relevante dentro del proceso educativo, es por eso por lo que deben utilizarse recursos didácticos que resulten atractivos para los alumnos (Valverde, Garrido, y Sosa, 2010).

Otra ventaja de los recursos digitales es la aportación del valor de la motivación, al generar ambientes de aprendizaje más agradables y dinámicos, así como posibilitar un mayor acercamiento con la realidad (Pinto, Gómez-Camarero, y Fernández-Ramos, 2012).

Internet es un medio que ofrece una gran diversidad de recursos digitales para un innumerable de utilidades posibles, los cuales, además, poseen características diferentes, por lo que ha sido necesario clasificarlos de acuerdo con el medio para el cual han sido creados. Para Cacheiro (2011) estos se clasifican en tres grupos, a saber:

a. Transmisivos, que son los que apoyan el envío, de manera efectiva, de mensajes del emisor a los 
Grace Liliana Figueroa Morán, Julio Pedro Paladines Morán, José Nevardo Paladines Morán...

destinarios.

b. Activos, que permiten que el aprendiz actúe sobre el objeto de estudio, y a partir de esta experiencia y reflexión, construya sus conocimientos.

c. Interactivos, cuyo objetivo es que el aprendizaje se dé a partir de un diálogo constructivo, sincrónico o asincrónico, entre individuos que usan medios digitales para comunicar e interactuar.

\section{Dimensiones para evaluar un recurso digital}

Ante esta clasificación, es importante poder tener en cuenta algunas dimensiones por las cuales los recursos digitales deben ser evaluados, estas fueron propuestas por Nokelainen (2006) y son:

1: Control del alumno (Nivel mínimo de memoria, unidades de contenido significativo, responsabilidad ante el propio aprendizaje).

2: Actividad del alumno (Razonamiento reflexivo, aprendizaje basado en problema, utilización de fuentes primarias, inmersión).

3: Aprendizaje cooperativo/colaborativo (Soporte para la conversación y el diálogo, trabajo en grupo, navegación síncrona y asíncrona).

4: Orientación objetivos (Objetivos explícitos, utilidad de los objetivos, orientación de resultados, feed-back pedagógico).

5: Aplicabilidad (Material auténtico, utilidad percibida, aprender haciendo, pre-testing y diagnóstico, incitación, unidades de contenido significativo).

6: Valor añadido (Para el aprendizaje, la efectividad, las imágenes, las animaciones).

7: Motivación (Orientación de objetivos intrínseca y extrínseca, significado de los estudios).

8: Valoración del conocimiento previo (Prerrequisitos, elaboración, ejemplos).

9: Flexibilidad (Pre-testing y diagnósticos, descomposición en tareas, tareas repetitivas).

10: Feed-back (Motivador, acertado, positivo).

\section{Recursos digitales utilizados actualmente}

Como se ha expresado anteriormente, existe una gran variedad de recursos digitales. Sin embargo, es conveniente considerar que estos mantengan una efectiva interacción entre el estudiante y el docente, y por ende que sirvan para facilitar la comprensión y apropiación de contenidos concretos. De tal manera que se detallan algunos recursos digitales utilizados actualmente (Cruz, 2017):

- TIC: Es muy común escuchar en la actualidad sobre el acelerado avance que han experimentado las TIC y la gran influencia que ejercen en todas las áreas del saber humano. Pero pese a este gran avance, algunas personas continúan dándole usos comunes que giran en torno a encontrar información, enviar y recibir correos electrónicos, hacer compras por Internet y disponer de aplicaciones que apoyen la actividad educativa.

- Bibliotecas digitales: Detrás de una biblioteca digital hay varias tecnologías que trabajan intermediando entre la biblioteca y los proveedores para ofrecer a los usuarios un acceso seguro y sencillo a todos los recursos que la componen.

- El entorno virtual consiste en plataformas en las cuales se lleva a cabo a la enseñanza y el aprendizaje, a través de un sistema estos recursos son parte representativa de la educación superior, ya que mediante esto se podría transmitir con más facilidad las enseñanzas brindadas por el docente a los alumnos ya que brinda fácil acceso a la información y a la comunicación. 


\section{Técnicas centradas en la individualización de la enseñanza}

La utilización de técnicas que se adaptan a las necesidades e intereses del estudiante, es decir, las herramientas que brinda el entorno permiten que se eleve la autonomía, el control del ritmo de enseñanza y las secuencias que marcan el aprendizaje. La utilización de estas técnicas requiere que el docente establezca una relación directa con el estudiante y asigne actividades en pro de su autorrealización y el grado de dificultad que así lo requiera, entre las que se tiene las siguientes (Fernández y González, 2015):

- Recuperación de información: el alumnado construye su propio conocimiento a través de la búsqueda y localización de información en Internet, y del análisis y valoración de la misma. Es importante que el docente intervenga en forma mínima, dando solamente las pautas de orientación necesarias para la actividad.

- Trabajo con materiales multimedia interactivos: esta modalidad consiste en el trabajo autónomo con materiales multimedia interactivos, algunos ejemplos son: los tutoriales, ejercicios y actividades prácticas, cuyo objetivo es la ejercitación del pensamiento crítico o del pensamiento creativo mediante métodos de análisis, ejercitación, solución de problemas o experimentación, el docente debe evaluar muy bien el material que le entregará al estudiante.

- Técnicas centradas en el pensamiento crítico: se pueden utilizar actividades para seleccionar y evaluar información o soluciones potenciales, así como la organización de esta. Pueden ser la creación de gráficos, ensayos sobre pros y contras, aspectos positivos y negativos, síntesis de lluvia de ideas, sumarios, reflexiones, esquemas, entre otros.

- Técnicas centradas en la creatividad: pretende motivar y potenciar la habilidad creativa de los estudiantes para la solución de un problema o situaciones, incitando la imaginación, la intuición, pensamiento metafórico, la elaboración de ideas, la curiosidad, implicación personal en la tarea, conexión con las experiencias previas, habilidad artística, búsqueda de problemas, entre otras.

- Técnicas expositivas y participación en gran grupo: Comunicación de uno al grupo: Estas técnicas parten de la construcción de conocimiento grupal a partir de información suministrada. Intervienen dos roles, el primero es del expositor que puede ser el docente, un experto o un estudiante y el segundo es el grupo receptor de la información.

- Otras técnicas: exposición didáctica, preguntas al grupo, simposio, mesa redonda o panel, entrevista o consulta pública, tutoría pública, tablón de anuncios y exposiciones.

\section{Herramientas de autor para elaboración de recursos digitales.}

Las herramientas de autor constituyen un conjunto de aplicaciones y software que proporciona directamente al usuario las soluciones necesarias para crear un contenido determinado (EducaRed, 2018). El contenido debe ser creado en función de sus objetivos, de su capacidad y competencia para hacerlo. La clasificación más pertinente y utilizada en relación con estos recursos, se realiza en función del tipo de productos (resultados) a los que se orienta (De la Torre y Violant, 2001).

\section{Ventajas de las herramientas de autor}

Las principales ventajas de estos sistemas son:

- Reducen el tiempo de desarrollo de aplicaciones hasta 1/8 del tiempo requerido con las formas de trabajos anteriores.

- Resultan más fáciles y rápidos de aprender que lenguajes de programación tradicionales.

- Al ser diseñados para un propósito específico, muchas de las necesidades más habituales de los creadores de software educativo han sido previstas de antemano y son fáciles de 
Grace Liliana Figueroa Morán, Julio Pedro Paladines Morán, José Nevardo Paladines Morán...

implantar.

- Mejora la calidad de enseñanza por parte del docente y del alumno.

- Ayuda a la valoración profesional del profesorado.

- Actualización de los métodos pedagógicos tanto en lo individual como la parte grupal.

Además, muchos de los programas de autor disponibles actualmente en el mercado son multiplataforma, es decir, son capaces de funcionar en distintos tipos de sistema operativo y ordenadores, facilitando así su utilización (Bautista, Martínez y Hiracheta, 2014).

\section{Algunas herramientas para crear actividades de aprendizaje.}

Dado que todo docente que utiliza la tecnología en su actividad utilizará una o varias herramientas de autor para generar los materiales concretos de la actividad didáctica (Delgado Fernández y Solano González, 2009). Se presentan a continuación algunas aplicaciones que podrían ser de gran ayuda.

- FreeMind. Creador de mapas mentales de carácter gratuito.

- CmapTools. de carácter gratuita, creada para elaborar mapas conceptuales.

- Create a Graph. Generador de gráficos que cuenta con una zona con herramientas específicas para niños.

- Graphmatica. Generador de gráficos de KSoft que cuenta con una versión gratuita.

- CadStd. Programa de tipo CAD (Diseño asistido por ordenador) con versión gratuita.

- LAMS (Learning Activity Management System). destinada al diseño, gestión y desarrollo de actividades de aprendizaje colaborativas y en línea.

- Hot Potatoes. creada para la generación de cuestionarios y test de diversos tipos y características. Inicialmente libre de carga para instituciones educativas y sin ánimo de lucro.

- JCLIC. permite al profesorado crear con facilidad recursos educativos digitales. Evolución del programa CLIC, lo cual ha traído una gran variedad de actividades, cuenta con nuevas funcionalidades y generar recursos cuya visualización no está restringida a ningún sistema operativo en particular.

- Squeak. Aplicación que funciona como un simulador de mundos virtuales donde es posible experimentar reproduciendo fenómenos y procesos de la realidad (o inventados). Se basa en la generación de objetos y guiones que les envían órdenes.

- Ardora. Aplicación para docentes, que les permite crear sus propias actividades, en formato HTML, de entre más de 45 tipos distintos tipos (crucigramas, sopas de letras, completar, paneles gráficos, relojes, etc.).

Los recursos digitales y su impacto en el desarrollo de la capacidad de análisis de los estudiantes universitarios.

\section{OBJETIVOS E HIPÓTESIS}

\section{Objetivo General}

O.G. Conocer el impacto de los recursos digitales en el desarrollo de la capacidad de análisis, de los estudiantes de primer semestre de la Carrera de Ingeniería en Computación y Redes en la asignatura de Introducción a la Informática. 


\section{Objetivos específicos emanados del anterior}

OE1: Analizar la importancia que tienen los recursos digitales como apoyo para el desarrollo de la capacidad de análisis en los estudiantes.

OE2: Identificar los recursos digitales que se pueden utilizar para promover el desarrollo de la capacidad de análisis de los estudiantes.

OE3: Utilizar recursos digitales en la labor educativa para promover el desarrollo de la capacidad de análisis de los estudiantes.

\section{Hipótesis}

Existirá una diferencia significativa entre los promedios de la capacidad de análisis de los estudiantes antes (pre-test) y después (post-test) de haber utilizado recursos digitales.

$\mathbf{H 0}=$ No hay una diferencia significativa de la capacidad de análisis de los estudiantes antes y después de la utilización de recursos digitales.

H1= Hay una diferencia significativa de la capacidad de análisis de los estudiantes antes y después de la utilización de recursos digitales.

\section{MATERIALES Y MÉTODOS \\ Participantes}

La muestra estuvo formada por 84 estudiantes de la carrera de Ingeniería en Computación y Redes. El grupo experimental estuvo compuesto por 51 estudiantes y el grupo de control por 33 estudiantes. La selección de los participantes fue por medio de un muestreo aleatorio. En la tabla 1 se puede observar la distribución por género y edad en cada uno de los grupos.

\section{Tabla 1}

Distribución por Género y Edad

\begin{tabular}{cccccc}
\hline & \multicolumn{2}{c}{ Género } & \multicolumn{3}{c}{ Edad } \\
\cline { 2 - 6 } & Hombre & Mujer & $\mathbf{1 7 - 1 9}$ & $\mathbf{2 0 - 2 2}$ & $>\mathbf{2 2}$ \\
\hline Experimental & 62,75 & 34,25 & 84,31 & 17,65 & 1,96 \\
\hline Control & 54,55 & 45,45 & 78,79 & 21,21 & 0 \\
\hline
\end{tabular}

\section{Instrumentos}

Se utilizaron varias herramientas de autor para valorar la capacidad de análisis, entre ellas: Jclic, Hot potatoes y Cmaptools, por ser aplicaciones gratuitas que permiten crear diversos recursos educativas y ofrecen retroalimentación inmediata (Rodríguez, 2015). Por otro lado, la herramienta Cmaptools brinda la posibilidad de construir y modificar mapas conceptuales de una manera sencilla, pero además permite a los usuarios colaborar a distancia en la construcción de sus mapas, publicarlos para que cualquier persona pueda acceder a ellos en Internet (Pontes, Serrano, Muñoz, y López, 2011). Las 2 primeras herramientas fueron utilizadas para desarrollar la capacidad de análisis a través de actividades interactivas online (preguntas cortas de opción múltiple sobre vídeos educativos; crucigramas, puzzle de intercambio, completar texto y sopa de letras).

La tercera herramienta fue empleada para evaluar de forma presencial la capacidad de análisis tanto para el grupo experimental como en el control. Consistió en elaborar un mapa conceptual y la socialización de este en el aula. 
Grace Liliana Figueroa Morán, Julio Pedro Paladines Morán, José Nevardo Paladines Morán...

\section{Procedimiento}

Este estudio sigue un diseño cuasi-experimental. Para ello, con 3 meses de anticipación se prepararon los materiales de medición de la capacidad de análisis, tanto para el pre-test como el post-test; de igual manera se hizo con los materiales interactivos online.

La medición pre-test se realizó en la segunda semana del semestre y la post-test en la duodécima semana. Durante las semanas comprendidas entre las administraciones del instrumento de recogida de datos, se aplicó el material interactivo online únicamente al grupo experimental.

La metodología docente utilizada en el grupo experimental incluyó clases magistrales, actividades prácticas y actividades interactivas online, mientras que para el grupo de control sólo se utilizaron clases magistrales y actividades prácticas.

\section{Análisis de Datos}

Para el análisis de los datos se utilizó Excel 2015 y se aplicó la prueba paramétrica t de Student, optimizando un valor de $\mathrm{p}<0,05$ para considerar significancia estadística. Se realizaron dos pruebas de $t$ de Student de diferencia de medias para muestras relacionadas, con el fin de comparar las diferencias entre las puntuaciones pre-test y post-test en ambos grupos.

\section{RESULTADOS}

En la tabla 2 y en la figura 1, se pueden observar las puntuaciones medias y desviaciones típicas en la capacidad de análisis tanto del grupo experimental como del control.

Los análisis muestran diferencias significativas para el grupo experimental $(\mathrm{t}=-3.56 ; \mathrm{p}=0,000)$ entre la puntuación obtenida en el pre-test $(\mathrm{M}=7,32$; $\mathrm{DT}=0,90)$ y en el post-test $(\mathrm{M}=7,90 ; \mathrm{DT}=$ $0,73)$; mientras que, en el grupo control no se muestran diferencias significativas $(\mathrm{t}=-1,61 ; \mathrm{p}=$ $0,111)$, las puntuaciones del pre-test fueron de $(M=7,25 ; \mathrm{DT}=0,75)$ y las del post-test $(\mathrm{M}=7,54$; $\mathrm{DT}=0,68)$.

\section{Tabla 2}

\section{Resultados de Capacidad de Análisis}

\begin{tabular}{ccc}
\hline & Pre-test & Post-test \\
\hline Experimental & $7,32(0,90)$ & $7,90(0,73)$ \\
\hline Control & $7,25(0,75)$ & $7,54(0,68)$ \\
\hline
\end{tabular}




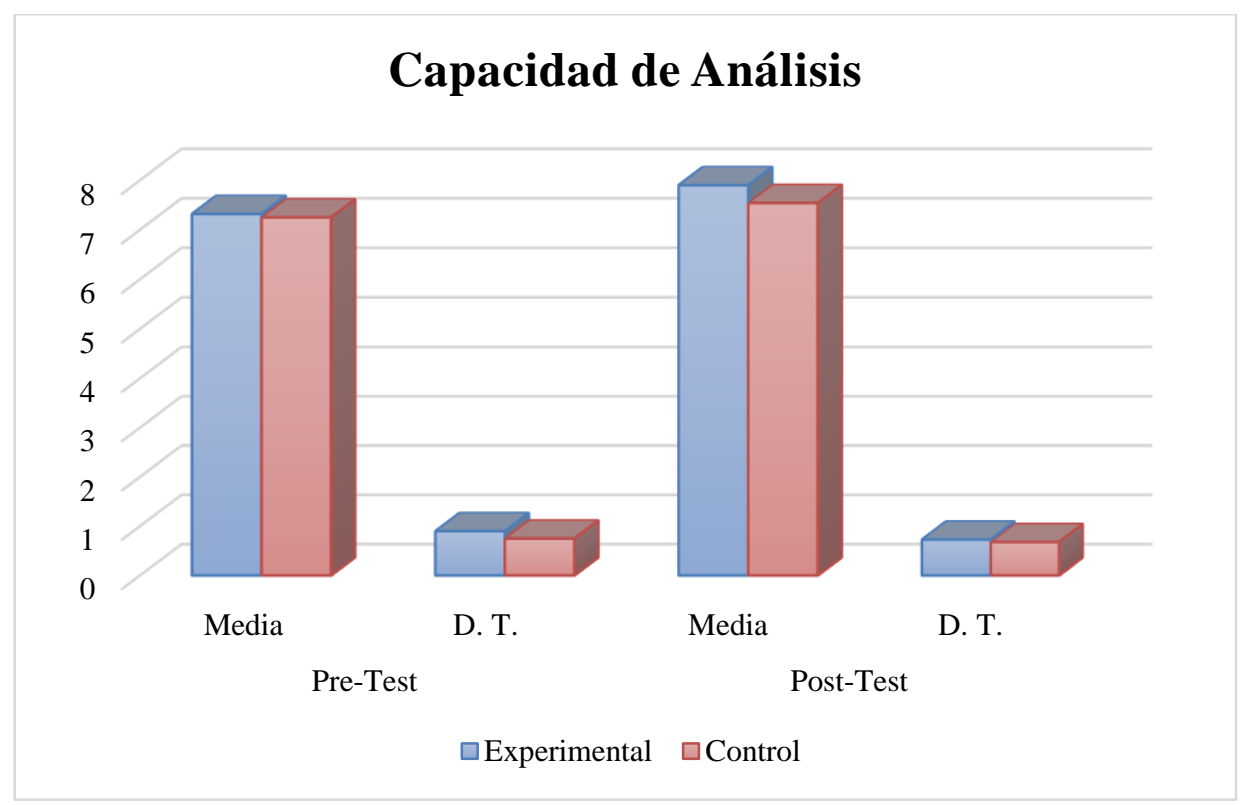

Figura 1. Capacidad de análisis: Estudio Descriptivo

Dado que el p valor en el grupo experimental es de 0,000 , se acepta la H1. Por tanto, se puede decir que, hay una diferencia significativa de la capacidad de análisis de los estudiantes antes y después de la utilización de recursos digitales.

\section{Discusión}

Se han llevado a cabo varios estudios relacionados con el uso de TIC como apoyo al proceso de enseñanza y aprendizaje (Colás, Ballesta, y De Pablos, 2018); sin embargo, existen pocos trabajos que se refieran el desarrollo de las capacidades específicas de los alumnos. En este trabajo se ha evaluado la capacidad de análisis de los estudiantes al inicio y al final del curso de la asignatura de Introducción a la Informática y se obtuvo un incremento significativo como se puede observar en la tabla 2, lo que lleva a considerar que la metodología utilizada en este proceso ha sido adecuada para el desarrollo de la competencia de análisis en los estudiantes.

\section{CONCLUSIONES}

La educación superior ha experimentado significativas transformaciones, el creciente número de estudiantes y su heterogeneidad; la multiplicación de sus esferas de actividad; la variedad de instituciones con fines, funciones y prioridades diferentes, son parte de las nuevas exigencias y desafíos a los que se enfrenta la educación superior.

En el contexto en el que se desarrollan los procesos de enseñanza y aprendizaje reclama dar respuesta a nuevos retos, donde habrá que validar el valor de las herramientas, los recursos y los métodos para educar a los estudiantes que necesitan comprender el sentido y el valor de su aprendizaje.

Las posibilidades que las TIC ofrecen para la enseñanza y la formación en diversas áreas son muy diversas y van desde facilitar la comunicación entre el profesor y los estudiantes hasta presentar información y desarrollar proyectos específicos para la gestión de información. La utilización de los recursos digitales en la actividad docente es una realidad creciente en la educación universitaria. Estos recursos deben ser eficaces y de calidad para que puedan ser 
Grace Liliana Figueroa Morán, Julio Pedro Paladines Morán, José Nevardo Paladines Morán...

utilizados por el profesorado en sus prácticas educativas.

Se demostró a través de la hipótesis planteada, que el uso de los recursos digitales genera un impacto significativo en el desarrollo de la capacidad de análisis de los estudiantes.

\section{REFERÉNCIAS BIBLIOGRÁFICAS}

Bautista, M., Martínez, A., y Hiracheta, R. (2014). El uso de material didáctico y las tecnologías de información y comunicación (TIC's) para mejorar el alcance académico. Ciencia y tecnología, 14, 183-194 Recuperado de http://www.palermo.edu/ingenieria/pdf2014/14/CyT_14_11.pdf.

Bautista, G.; Borges, F.; Forés, A. (2006). Didáctica universitaria en entornos virtuales de enseñanza-aprendizaje. 13, Madrid, España: Narcea Ediciones,. Recuperado de http://www.terras.edu.ar/biblioteca/2/2BAUTISTAGuillermo-BORGES-Federico-FORES-AnnaCAP2Ser-estudiantes-en-entornos-virtuales.pdf.

Cacheiro, M. (2011). Recursos Educativos TIC de información, colaboración y aprendizaje. Pixel BIt, Revista de Medios y Educación, 69-81.

Clutterbuck, D. (2015). Mentoring: Técnicas para motivar, desarrollar las relaciones, potenciar el talento y mejorar la productividad. España: Profit Editorial.

Colás , M., Ballesta, J., y De Pablos, J. (2018). Incidencia de las TIC en la enseñanza en el sistema educativo español: una revisión de la investigación. Revista de Educación a Distancia. 56, DOI: http://dx.doi.org/10.6018/red/56/2 Recuperado de http://www.um.es/ead/red/56/colas_et_al.pdf.

Cruz, L. M. (2017). Tecnologías digitales y docencia, prácticas de profesores. Estudio cualitativo en la universidad pedagógica nacional. Revista Latinoamericana de Ciencias de la Comunicación, 14(26).74-85. Recuperado de https://www.alaic.org/revista/index.php/alaic/article/view/916/471

De la Torre, S., \& Violant, V. (2001). Estrategias creativas en la enseñanza universitaria. Creatividad y Sociedad, 3, 10-35.

Delgado Fernández, M., y Solano González, A. (2009). Estrategias didácticas creativas en entornos virtuales para el aprendizaje. Revista Electrónica "Actualidades Investigativas en Educación", 9(2). 1-21. Recuperado de http://www.redalyc.org/articulo.oa?id=44713058027

EducaRed. (12 de mayo de 2018). Obtenido de Conocimiento con todos y para todos: Recuperado de https://www.ecured.cu/Herramientas_de_autor

Fernández, M. D., y González, A. S. (2015). Estrategias didácticas creativas en entornos virtuales para el aprendizaje/Creative didactic strategies in virtual surroundings for the learning. Actualidades Investigativas en Educación, 9(2).doi: $\quad$ HYPERLINK "https://doi.org/10.15517/aie.v9i2.9521" https://doi.org/10.15517/aie.v9i2.9521 .

Isusi, R. (2018) Innovación, Interdisciplinariedad y Educación Artística en la formación docente universitaria. DEDiCA. Revista de Educação E Humanidades, 13, 43-53. ISSN: 2182018X

Medina, A., y Ballano, S. (2015). Retos y problemáticas de la introducción de la educación mediática en los centros de secundaria. Revista de Educación, 135-158.

Mondragón, C. A. (2014). Las Tecnologías de la Información y la Comunicación (TIC) y la subjetividad local. El giro de la identidad juvenil. Plumilla Educativa, 14, 173-189.

Nokelainen, P. (2006). An empirical assessment of pedagogical usability criteria for digital learning material with elementary school students. Educational Technology And Society, 178 - 197. Recuperado de https://pdfs.semanticscholar.org/ea96/b628f440642d72026c14710a67ccd06f41f1.pdf.

Pontes, A., Serrano, R., Muñoz, J., y López, I. (2011). Innovación educativa sobre aprendizaje colaborativo con CmapTools en la formación inicial docente. Revista Iberoamericana de Evaluación Educativa, 4(2), 136-154.

Pinto, M., Gómez-Camarero, C., y Fernández-Ramos, A. (2012). Los recursos educativos electrónicos: perspectivas y herramientas de evaluación. Perspectivas en Ciencia de la Información, 17(3), 82-89. doi: 10.1590/S141399362012000300007.

Salinas, J. (2004). Innovación docente y uso de las TIC en la enseñanza universitaria. Revista Universidad y Sociedad del Conocimiento, 1 (1). Recuperado de http://www.redalyc.org/pdf/780/78011256006.pdf.

Salinas Ibánez, J. (2004). Cambios metodológicos con las TIC. Estrategias didácticas y entornos virtuales de enseñanza-aprendizaje. Bordón Revista de Pedagogía, 56(3-4), 469-481. 
UNESUM-Ciencias: Revista Científica Multidisciplinaria

ISSN 2602-8166

Los Recursos Digitales y su Impacto en el desarrollo de la Capacidad de Análisis de los estudiantes Universitario

Trigueros, F., Sánchez, R., \& Vera, M. (2012). El profesorado de educación primaria ante las TIC: realidad y retos. Revista electrónica Interuniversitaria de Formación del Profesorado., 15(1), 101-112. Recuperado de http://www.aufop.com/aufop/uploaded_files/articulos/1335399123.pdf.

Valverde, J. , Arroyo, M. y Sosa, M. (2010). Políticas educativas para la integración de las TIC en Extremadura y sus efectos sobre la innovación didáctica y el proceso enseñanza-aprendizaje: la percepción del profesorado. Revista de Educación, 352, 99-124.

Rodríguez (2015). Uso de Software Educativos y objetos virtuales de Aprendizaje para motivar la formación en ciencias básicas. Repositorio Digital de la Universidad Tecnológica de Pereira, Recuperado de http://repositorio.utp.edu.co/dspace/bitstream/handle/11059/6067/371334R696.pdf?sequence=1.

Zapata, M. (2012). Recursos educativos digitales: conceptos básicos. Obtenido de Programa Integración de Tecnologías, Universidad de Antioquia. Recuperado de http://aprendeenlinea.udea.edu.co/boa/contenidos.php/d211b52ee1441a30b59ae008e2d31386/845/estilo/aHR0cDov L2FwcmVuZGVlbmxpbmVhLnVkZWEuZWR1LmNvL2VzdGlsb3MvYXp1bF9jb3Jwb3JhdGl2by5jc3M=/1/conten ido/. 
Grace Liliana Figueroa Morán, Julio Pedro Paladines Morán, José Nevardo Paladines Morán...

54 UNESUM-Ciencias. Publicación cuatrimestral. Vol. 4, Año 2020, No. 1 (Enero - Abril) 